\title{
La mémoire et l'oubli dans les œuvres de Shakespeare
}

\author{
Henri Suhamy \\ Christophe Hausermann (éd.)
}

\section{(2) OpenEdition \\ Journals}

Édition électronique

URL : http://journals.openedition.org/shakespeare/1912

DOI : 10.4000/shakespeare.1912

ISSN : 2271-6424

Éditeur

Société Française Shakespeare

Édition imprimée

Date de publication : 1 avril 2013

Pagination : 1-9

ISBN : 2-9521475-9-0

Référence électronique

Henri Suhamy, "La mémoire et l'oubli dans les œuvres de Shakespeare », Actes des congrès de la Société française Shakespeare [En ligne], 30 | 2013, mis en ligne le 03 avril 2013, consulté le 20 avril 2019. URL : http://journals.openedition.org/shakespeare/1912 ; DOI : 10.4000/shakespeare.1912 


\section{Shakespeare et la mémoire}

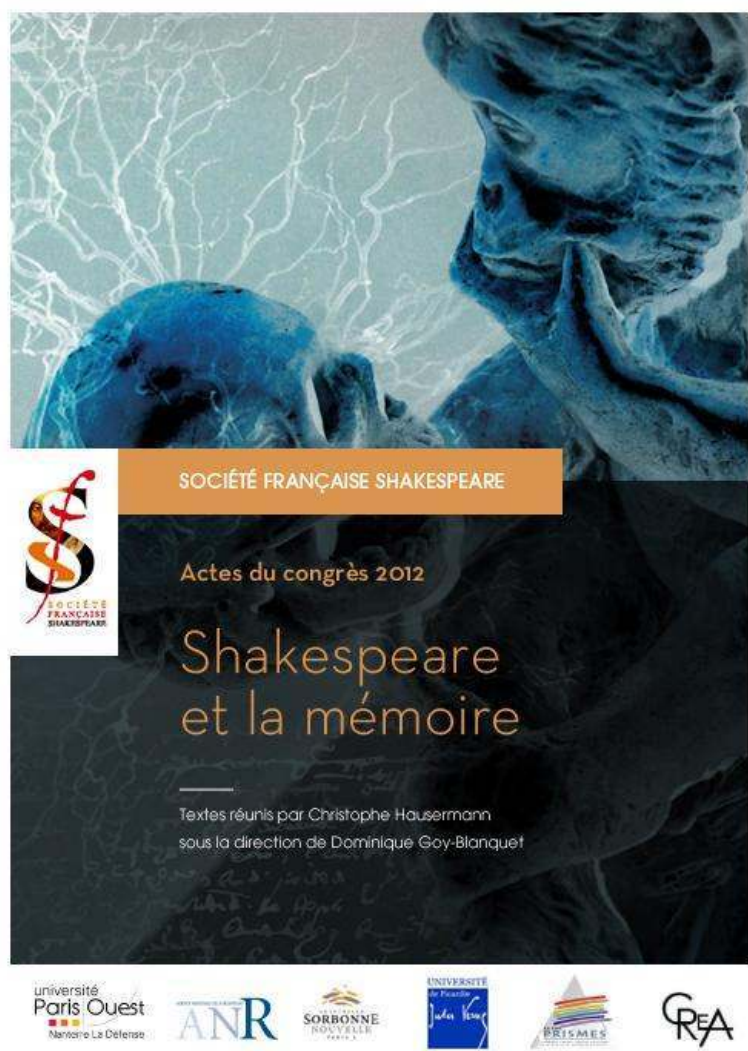

actes du Congrès

organisé par la

SOCIÉTÉ FRANÇAISE SHAKESPEARE

les 22, 23 et 24 mars 2012

textes réunis par

Christophe HAUSERMANN

sous la direction de

Dominique GoY-BLANQUET 


\section{COUVERTURE}

d'après l'affiche de Claire Colombet

conception graphique et logo

Pierre Kapitaniak

mise en page et corrections

Christophe Hausermann

(C) 2012 Société Française Shakespeare

Institut du Monde Anglophone

Université de Paris III - Sorbonne Nouvelle

5 rue de l'École de Médecine 75006 Paris

www.societefrancaiseshakespeare.org

Tous droits de traduction, de reproduction et d'adaptation réservés pour tous les pays 


\title{
LA MEMOIRE ET L'OUBLI DANS LES EUVRES DE SHAKESPEARE
}

\author{
Henri SUHAMY
}

\begin{abstract}
Les mots se référant à la mémoire et à l'oubli reviennent fréquemment dans les œuvres de Shakespeare, mais le premier souvenir de ce thème du souvenir qui vient à la mémoire du lecteur est le Sonnet 30 , où se trouve l'expression par laquelle Scott Moncrieff a intitulé sa traduction de Proust: Remembrance of things past. Ce thème lié au devoir de deuil, ainsi qu'à des situations impliquant l'honneur, la loyauté, le serment, la généalogie et le patrimoine aristocratiques, sert de ressort rhétorique, dramatique et émotif dans les œuvres théâtrales, où il s'oppose à l'oubli, qui implique infidélité, parjure et reniement. La mémoire sert aussi d'accessoire dramaturgique, car l'action théâtrale a besoin de récits rétrospectifs. Enfin Shakespeare soulève la question de la pérennité de la création littéraire dans la mémoire des hommes, et prophétise la survie de son œuvre.
\end{abstract}

Words referring to memory and forgetfulness are frequent in Shakespeare, but what first occurs to the reader's own memory, as regards the memory motif, is Sonnet 30, which contains the phrase used by Scott Moncrieff as a title to his translation of Proust's novel: Remembrance of Things Past. This theme, associated with the duty of mourning and remembering the dead, and also to situations implying honour, loyalty, oaths, aristocratic genealogy and moral heirlooms, is used on the stage as a rhetorical, dramatic and emotional mainspring, in opposition to oblivion, implying unfaithfulness, perjury and apostasy. Memory also belongs to the stock-in-trade of dramaturgy, for the action of a play needs retrospective narrations. Last but not least, Shakespeare raises the question of the perenniality of literary creation in men's memories, prophesying the survival of his own works.

$\mathrm{D}$ e nos jours les machines aussi sont dotées de mémoire, et les puces en ont plus que les éléphants, les puces électroniques, bien entendu. Il n'est plus nécessaire de s'encombrer des anciennes et volumineuses concordances pour apprendre que les œuvres de Shakespeare, tous genres confondus, contiennent 67 occurrences du mot memory. Plus fréquents encore sont les emplois de remember, auquel s'ajoutent les désinences de la conjugaison, et remembrance, qui ensemble atteignent le nombre de 316 occurrences. Opposé à la mémoire et aux souvenirs se trouve forcément le thème de l'oubli, moins représenté statistiquement, mais qui atteint, entre oblivion, oblivious, forget, forgetful, forgetfulness, le nombre de 136 occurrences. Si on ajoute les mots qui font partie des champs sémantiques de l'ingratitude, de l'infidélité, du reniement, du parjure, volontaire ou involontaire, la quantité augmente. Ces recensions numériques ne donnent qu'une idée abstraite, sans substance, de l'importance de ces thèmes dans la pensée de Shakespeare. Il vaut peut-être mieux, avant de s'en tenir aux usuels ou aux accessoires mécaniques qui font à notre place le travail de mémoire, se fier à la 
nôtre, de mémoire, en premier lieu. Or quel est le souvenir de ce thème du souvenir qui vient immédiatement à l'esprit ? Inévitablement le Sonnet 30, qui commence ainsi :

When to the sessions of sweet silent thought

I summon up remembrance of things past $[\ldots]^{1}$

De plus ces deux vers évoquent un autre souvenir, celui du titre emprunté à Shakespeare et donné dès 1922 par l'écrivain écossais Scott Moncrieff à sa traduction du grand roman de Proust : Remembrance of Things Past. Or il y a dans le premier vers du sonnet un mot inattendu qui constitue un pré-écho, comme un commentaire par anticipation de l'œuvre de Proust, l'adjectif sweet. Convoquer ses souvenirs dans le silence solitaire de la pensée, les remâcher, les fixer sur l'écran du cerveau, cela est donc délicieux. William Shakespeare lui-même et ses personnages n’ont-ils jamais été hantés par leur mémoire, accablés, persécutés par le remords et par les regrets, étouffés par le reflux nauséeux du passé, à la manière de ceux de Eugene O'Neill, dont certains semblent n'avoir d'autre aspiration que de se plonger au plus vite dans l'eau du Léthé ? On pense forcément au couple Macbeth, au Cardinal Beaufort dans la seconde partie d'Henry VI, au roi Jean ou même à Richard III, tout endurci qu'il est, à Henry IV, qui remet toujours à plus tard son projet d'expier le meurtre de Richard II et d'en exorciser la mémoire lancinante par un pèlerinage en Terre Sainte. Quelquefois ce sont non les mauvaises actions mais les malheurs passés qui oblitèrent le goût de vivre, poussant Ægéon ou Périclès à une errance désespérée, à "sans amour s'en aller sur la mer », comme dit Théophile Gautier ${ }^{2}$. Cependant si les actions passées laissent des obsessions qu'on aimerait pouvoir chasser, il existe des souvenirs qu'on éprouve le besoin de ramener à la conscience, comme ceux qu'annoncent les deux premiers vers du Sonnet 30, et dont on attend un certain plaisir. Pourtant dans ce cas on se heurte à une péripétie inattendue. En effet dans la suite du poème, il n'est pas question d'une épiphanie à la Joyce ou à la Proust, d'une madeleine dont la réminiscence gustative provoque une extase quasi hallucinatoire, ou des pavés de Venise gravés dans la mémoire non seulement par la

\footnotetext{
${ }^{1}$ The Sonnets, in The Oxford Shakespeare, ed. Stanley Wells and Gary Taylor, Oxford, O.U.P, $2^{\text {nd }}$ edition, 2005
}

2 Dans La Comédie de la mort (1838). 
vision mais aussi par la perception synesthésique du corps tout entier 3 . Ce sont plutôt des souvenirs douloureux que Shakespeare évoque, et même convoque, puisqu'il file une métaphore judiciaire pour introduire son thème. De plus le mot sessions implique l'idée d'une citation à comparaître à intervalles réguliers, au moins une fois par an. Il s'agit de célébrer ce qu'on appelle par métonymie la mémoire, c'està-dire le souvenir des amis morts, des proches aussi car le mot friends, dans l'anglais élisabéthain, et jusqu'au $\mathrm{XIX}^{\mathrm{e}}$ siècle, désigne assez souvent les membres de la parentèle.

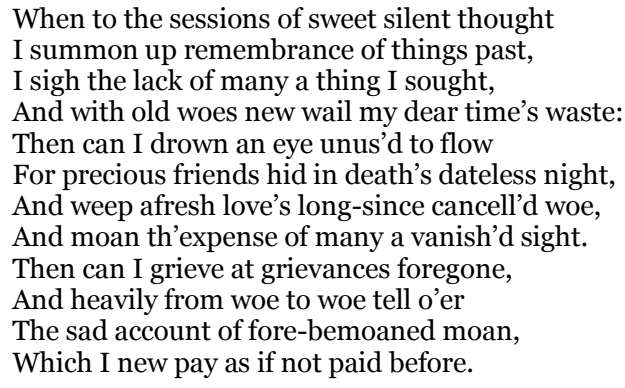

When to the sessions of sweet silent thought I summon up remembrance of things past,

On peut se demander comment Shakespeare a pu accoler l'adjectif sweet à la litanie funèbre qui allait suivre, mais on parle parfois de douleur exquise, selon un cliché en forme d'oxymore, et la poésie ellemême, forcément présente, a le don de tout transfigurer, de tout enchanter. Shakespeare connaissait le célèbre vers de Virgile, «Infandum regina jubes renovare dolorem 4 », puisqu'il y fait allusion dans The Comedy of Errors :

A heavier task could not have been imposed

Than I to speak my griefs unspeakable.

$($ I.i.31-2) 5

Cela n'empêche pas Ægéon de broder complaisamment sur ses malheurs passés, ce qui est à la fois poétiquement et dramatiquement nécessaire dans une scène d'exposition. Le théâtre ne peut guère se passer de récits rétrospectifs, même et surtout quand la pièce commence in medias res. La mémoire fait donc partie des accessoires

\footnotetext{
3 Cf. John Ruskin, The Stones of Venice, 3 vols.,1851-1853.

4 « Reine, vous m'ordonnez de rouvrir de cruelles blessures. », Virgile, Énéide, liv. II, v. 3.

5 The Comedy of Errors, in The Oxford Shakespeare, op. cit.
} 
indispensables de la dramaturgie. Comme ses sœurs, Melpomène, muse de l'harmonie devenue celle de la tragédie, est bien la fille de Mnémosyne. Mais le sonnet 30 ne fait pas partie des œuvres théâtrales, de plus la mémoire y est traitée en tant que thème explicite, elle ne constitue pas le support implicite des récits qui fournissent au public des données sur ce qui se situe dans le temps en amont du drame. Dans le sonnet, la mémoire constitue un devoir, le devoir de deuil, même si le poème se termine par une pirouette annonçant le retour empressé à la vie et à l'amour. Incidemment le Sonnet 71 (No longer mourn for me when I am dead) semble prendre le contre-pied du précédent, mais il constitue une épitrope, c'est-à-dire une injonction ironique, non dénuée d'amertume. La remémoration, qui prend parfois la forme d'une commémoration, constitue donc une tâche qu'il faut accomplir. La mémoire n'est donc pas seulement une faculté mentale qui permet aux hommes d'avoir une vie intérieure, de rester propriétaires de leur expérience passée, d'échapper à la fluidité destructrice du temps, et dont la perte, notamment pour cause de sénilité, est «In second childishness and mere oblivion,» (As You Like It, II.vii.166) constituant un malheur irrémédiable. Comme tous les devoirs, il exige un effort. Incidemment on peut rappeler que pour un acteur professionnel comme l'était notre auteur, la nécessité d'apprendre des textes par cœur implique aussi un autre type d'effort, non pas celui de la volonté de surmonter des blocages psychologiques, ou le besoin instinctif qui nous pousse à préférer vivre dans l'instant et dans l'espérance d'un avenir heureux plutôt que dans la réminiscence du passé, mais celui des facultés mnémotechniques. Voir un autre sonnet, le vingt-troisième, qui commence par l'allusion à un acteur malhabile dont le trac inhibe la mémoire, ou dont les défaillances de la mémoire provoquent le trac.

Il est vrai aussi que les hommes sont pleins de contradictions, ils oublient le passé et pourtant l'idéalisent. Tel Don Quichotte, ils rêvent d'un retour à la chevalerie, nostalgiques d'un âge d'or qui n'a sans doute jamais existé. La figure du laudator temporis acti ${ }^{6}$ n'existe pas seulement parmi les vieillards sur le point de mourir, comme Jean de Gand qui regrette l'époque des Croisades, quand ses compatriotes aujourd'hui décadents étaient loin de chez eux, renommés pour leur service chrétien et leur vraie chevalerie (Richard II, II.i.54). Le jeune

\footnotetext{
6 « Faisant l'éloge du temps passé », Horace, Art poétique, vers 178.
} 
Orlando, dans As You Like It, fait l'éloge du temps passé, quand les domestiques servaient leurs maîtres avec désintéressement et loyauté (II.iii.57-63).

Revenons au devoir moral, aux cérémonies du souvenir, thèmes nullement absent des œuvres dramatiques, où ils constituent un motif récurrent. La trilogie d'Henry VI commence par une oraison funèbre en l'honneur du défunt roi Henry V. Hamlet, qui dans le texte de la pièce sinon sur les scènes actuelles, porte des habits de deuil, s'indigne de l'oubli dont on recouvre les morts, le roi son père particulièrement, mais sa véhémence sermonneuse s'étend à tous les morts. Plus tard Laertes reproche à Claudius non seulement d'avoir bâclé le cérémonial des obsèques, mais aussi de ne pas avoir orné la tombe de son père des trophées rapportés des combats, et autres objets et inscriptions destinés à lui rendre un hommage posthume et durable (IV.v.210-2). Pour la collectivité, incapable peut-être de se livrer aux méditations intérieures évoquées par Shakespeare dans le Sonnet 30, le culte des morts, comme tous les cultes, a besoin de rites, de signes visibles, d'icônes. Le souvenir de Roméo et de Juliette est pérennisé par une statue en or massif, Octave César promet de faire célébrer des funérailles spectaculaires en l'honneur d'Antoine et de Cléopâtre, qui étaient pourtant ses ennemis. Dans l'un de ses sarcasmes amers et agressifs, Hamlet dit que pour qu'on se souvienne de lui pendant plus de six mois après sa mort, un grand homme doit construire des églises :

there's hope a great man's memory may outlive his life half a year. But, by'r Lady, a must build churches then,

(III.ii.125-126)

Le thème de la commémoration a une incidence morale et psychologique, allant jusqu'à constituer un ressort dramatique, car les personnages qui se posent en donneurs de leçons font pression sur d'autres personnages en leur rappelant le devoir de mémoire et de fidélité, le culte des ancêtres, le respect de la parole donnée. Dans Julius Caesar ce rappel des devoirs de mémoire envers les ancêtres et l'idée qu'ils ont laissé un patrimoine de valeurs intangibles à se transmettre de génération en génération sert de levier à Cassius pour convaincre Brutus de réactualiser l'histoire ancienne, d'entrer dans une conspiration qui répète celle du premier des Brutus. On sent l'ombre de ce personnage à la fois réel et légendaire peser sur tout le discours de Cassius, et il surgit en personne dans la péroraison : 
O you and I have heard our fathers say

There was a Brutus once that would have brook'd

The eternal devil to keep his state in Rome

As easily as a king.

Brutus prend sa revanche sur son dangereux ami au cours de la querelle du quatrième acte. Il retourne sur lui l'impératif catégorique de la remémoration par une injonction en forme de chiasme légèrement tronqué qui claque d'une manière mordante et péremptoire : "Remember March, the ides of March remember! » (Iv.iii.16-18).

La véhémence de ces tirades et de ces dialogues conflictuels soulève une question difficile qui se trouve au cœur de l'œuvre shakespearienne et qui ne recevra sans doute jamais de réponse consensuelle. Comment le public est-il censé réagir devant de tels déchaînements de passion issus de convictions enracinées dans la mentalité collective ? La dialectique propre au théâtre peut conduire à relativiser les dogmes, à juger Cassius comme le scélérat de l'histoire, ou au moins comme un tentateur qui exerce sur Brutus un chantage moral et idéologique pour arriver à ses fins. Mais ce jugement ne tient pas compte du fait que Cassius, "le dernier de tous les Romains » c'est-à-dire le dernier représentant d'un passé glorieux et regrettablement révolu, comme l'appelle Brutus lui-même dans son oraison funèbre (v.iii.99), n'est pas vraiment un hypocrite, ni un pervertisseur d'idéaux, même si sa rhétorique apparait comme trop habile pour être honnête. Il croit à ce qu'il dit, et le public du théâtre ne reste pas insensible à son éloquence, fondée sur un système de valeurs auquel adhèrent les spectateurs eux-mêmes, tout en se sentant coupables de ne pas souvent le mettre en pratique. Entre la scène et la salle un courant passe, l'exaltation du passé, le respect de l'héritage moral ou social laissé par les anciens de la tribu ne laissent personne indifférent. Il existe aussi, dans la société encore très visiblement hiérarchisée où vivait Shakespeare, une ethnologie de la mémoire. Elle se trouve à la base de l'idéologie aristocratique et de l'exaltation de l'honneur en tant que valeur morale et héréditaire, dont on trouve des empreintes profondes dans le texte shakespearien, comme plus tard chez Lope de Vega, Calderón, Corneille et bien d'autres. Les nobles ont une dimension dont les gens du commun sont privés, une mémoire qui 
peut remonter très loin dans le passé et que matérialise un arbre généalogique bien en vue.

Dans Henry $V$, l'évêque d'Ely pousse le roi à entrer en guerre en évoquant le souvenir épique de son arrière-grand-père Édouard III et de ses compagnons :

Awake remembrance of these valiant dead,

And with your puissant arm renew their feats.

(I.ii.115)

Plus tard, dans l'exhortation d'Azincourt, le roi lui-même, s'instaurant comme le porte-parole de la mémoire collective, anticipe sur la commémoration des exploits non encore accomplis ; il investit déjà le futur proche du prestige d'un passé lointain :

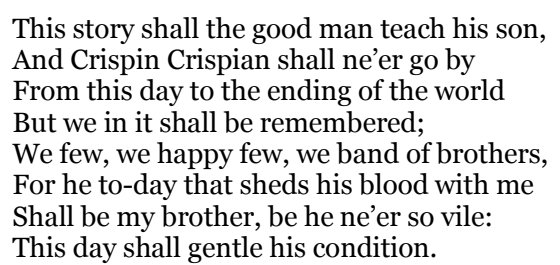

On peut se demander si le vrai Henri $\mathrm{V}$ a conféré un titre de noblesse à tous les archers et à tous les fantassins d'Azincourt, ainsi qu'à leur descendance, rendue alors dépositaire d'un souvenir glorieux. S’il ne l'a pas fait, il a oublié sa promesse, et ainsi a failli au devoir de mémoire, mais la promesse elle-même a probablement été inventée rétrospectivement par Shakespeare. Comme quoi l'imagination se plait parfois et même souvent à enjoliver la mémoire, d'où la métamorphose de l'Histoire en légende.

L'oubli implique la perte de l'honneur, et aussi la perte de l'amour, qui dans la vision idéalisée qu'en offre la poésie aussi bien que le théâtre de Shakespeare, implique la constance. C'est ce qu'affirme Protée lui-même dans The Two Gentlemen of Verona après avoir reconnu l'oubliée Julia sous les traits de Sébastien, qui loin de son cœur, n'est pourtant pas loin de ses yeux. L'amnésie amoureuse provoque l'aveuglement. Il tire de l'aveu de son infidélité une conclusion morale, quasi philosophique ou théologique. 
O heaven, were man

But constant, he were perfect. That one error

Fills him with faults, makes him run through all th'sins.

(V.iv.109-11)

S'il est vrai que les hommes méritent d'être blâmés pour les carences de leur mémoire et de leur fidélité, il faut se montrer indulgent envers eux, c'est-à-dire envers nous-mêmes, car le péché d'oubli n'est pas seulement dû à la faiblesse physique et morale des êtres humains. Ils vivent dans le temps créateur et destructeur de tout ce qui existe, le temps, leitmotiv récurrent dans les œuvres et dans la pensée de Shakespeare, qui l'a fait apparaître en personne dans The Winter's Tale, sous sa forme allégorique et investi de la fonction du chœur. Il s'y montre assez débonnaire, il accepte pour une fois de réparer les irréparables outrages pour lesquels il est tristement réputé, car même les monuments commémoratifs subissent son érosion. Lutter contre le temps confère un certain héroïsme à ceux qui font l'effort de ne rien oublier, de convoquer leurs souvenirs, même les plus douloureux, et qui en plus travaillent à leur propre survie dans la mémoire des hommes. C'est le cas des artistes et des poètes, de telle sorte que leurs œuvres s'installent dans notre esprit, font partie de notre expérience et de notre vie. Après le sonnet 30, le sonnet 55 commence ainsi :

Not marble, nor the gilded monument[s]

Of princes shall outlive this powerful rime,

Pourquoi, après avoir écrit ces vers, Shakespeare ne s'est-il pas préoccupé, semble-t-il, de publier ses sonnets ? On peut se livrer à des conjectures, à condition de ne pas les transformer en certitudes. On peut aussi remarquer que du temps d'Horace, auteur de la fameuse ode exegi monumentum aere perennius (j'ai édifié un monument plus pérenne que le bronze) dont Shakespeare semble s'être inspiré, l'imprimerie n'existait pas. On écrivait alors sur des plaques de cire, substance notoirement moins durable que le bronze et même que le papier. Pourtant le non omnis moriar 7 d'Horace s'est révélé prophétique : ses poèmes restent dans la mémoire des hommes, tout comme les œuvres de Shakespeare, dont certaines ont en même temps

7 « Je ne mourrai pas tout entier », Horace (ode xxiv, liv. III, vers 6). 
pour mission de perpétuer la mémoire historique, comme l'annonce Cassius dans Julius Caesar :

How many ages hence

Shall this our lofty scene be acted over,

In states unborn and accents yet unknown!

(III.i.111-3)

C'est ce travail de mémoire, de transmission vivante, non dévotieusement rituelle et bureaucratique, mais sentie comme allant de soi, en plus des thèmes que j'ai brièvement et sommairement abordés, que les participants à ce congrès ont éclairés et développés. Je leur laisse la parole.

Henri SUHAMY

Université Paris Ouest Nanterre La Défense 